\title{
sciendo
}

Moroccan J. of Pure and Appl. Anal. (MJPAA)

Volume 4(2), 2018, Pages 62-76

ISSN: Online 2351-8227 - Print 2605-6364

DOI 10.1515/mjpaa-2018-0007

\section{Weighted Variable Exponent Sobolev spaces on metric measure spaces}

\author{
Moulay Cherif Hassib ${ }^{1, a}$ ANd Youssef AKdim ${ }^{2, b}$
}

\begin{abstract}
Aвstract. In this article we define the weighted variable exponent-Sobolev spaces on arbitrary metric spaces, with finite diameter and equipped with finite, positive Borel regular outer measure. We employ a Hajlasz definition, which uses a point wise maximal inequality. We prove that these spaces are Banach, that the Poincaré inequality holds and that lipschitz functions are dense. We develop a capacity theory based on these spaces. We study basic properties of capacity and several convergence results. As an application, we prove that each weighted variable exponent-Sobolev function has a quasi-continuous representative, we study different definitions of the first order weighted variable exponent-Sobolev spaces with zero boundary values, we define the Dirichlet energy and we prove that it has a minimizer in the weighted variable exponent -Sobolev spaces case.
\end{abstract}

2010 Mathematics Subject Classification. Primary: 46E35; 31B15; 46E30; 42B25; 28A80.

Key words and phrases. weighted variable exponent Sobolev spaces on metric spaces, capacity, weighted variable exponent Sobolev with zero boundary values, Dirichlet energy.

\section{Introduction}

The theory of Sobolev space was originally developed in the domains of $\mathbb{R}^{N}$ using the notion of distributional derivatives. To generalize this theory to metric spaces, P. Hajlasz showed in [7] that a p-integrable function $u$, $1<p<\infty$, belongs to $W^{1, p}\left(\mathbb{R}^{N}\right)$ if and only if there exists a non-negative p-integrable function $g$ such that

$$
|u(x)-u(y)| \leqslant|x-y||g(x)+g(y)|
$$

Received February 6, 2019 - Accepted April 3, 2019.

(C) The Author(s) 2017. This article is published with open access by Sidi Mohamed Ben Abdallah University.

${ }^{1}$ University Sidi Mohamed Ben Abdellah, Faculty of science and technique, Fez, Laboratory: LSI, Taza, Morocco.

${ }^{2}$ University Sidi Mohamed Ben Abdellah, Faculty of science Dhar El Mahraz, Laboratory: LAMA Fez, Morocco.

${ }^{a}$ e-mail: cherif_hassib@yahoo.fr

${ }^{b}$ e-mail: akdimyoussef@yahoo.fr. 
for almost every $x, y \in \mathbb{R}^{n}$. This inequality can be stated also in metric measure spaces if $|x-y|$ is replaced by the distance between the points $x$ and $y$.

This theory was generalized by N. Aissaoui in [4] in the Orlicz-Sobolev spaces; by Petteri Harjulehto, Peter Hasto Mikko Pere in [15] in the variable exponent spaces, by Takao Ohno and Tetsu Shimomura in [16] in the Musielak-Orlicz-Sobolev spaces and by M.C. Hassib, Y. Akdim, N. Aissaoui in [8] in the Musielak-Orlicz-Sobolev spaces also and by using another method. The objective of this paper is to develop this theory in the setting of weighted variable exponent -Sobolev spaces.

The paper is organized as follows:

In section 1 we recall some important definition and some results of weighted variable exponent spaces $L^{p(.)}(\Omega, \omega)$, in section 2, we define the weighted variable exponent -Sobolev spaces on metric space, we show that: it is a Banach space, the Poincaré inequality holds and lipschitz continuous functions are dense. In section 3, we develop a capacity theory based on this space, we study basic properties of capacity, including monotonicity, countable subadditivity and several convergence results are studied. As an application we prove that each weighted variable exponent-Sobolev function has a quasi-continuous representative. In section 4 we study different definitions of the first order weighted variable exponent-Sobolev spaces with zero boundary values, we define the Dirichlet energy and we prove that it has a minimizer in the weighted variable exponent -Sobolev spaces on metric measure space case.

\section{Preliminary}

2.1. Variable exponent Lebesgue spaces on metric measure spaces.[9]. By a metric measure space we mean a triple $(X, d, \mu)$, where $X$ is a set, $d$ is a metric on $X$ and $\mu$ is a non-negative Borel regular outer measure on $X$ which is finite in every bounded set. For simplicity, we often write $X$ instead of $(X, d, \mu)$. For $x \in X$ and $r \geqslant 0$ We call a measurable function $p: X \longrightarrow[1,+\infty)$ a variable exponent.

For $A \subset X$ we define $p_{A}^{+}=e s s \sup _{x \in A} p(x)$ and $p_{A}^{-}=e s s \inf _{x \in A} p(x)$. we use the abbreviations $p^{+}=p_{X}^{+}$and $p^{-}=p_{X}^{-}$.

We denote

$$
C_{+}(X)=\left\{\text { continuous function } p(\cdot): X \longrightarrow[1,+\infty) / 1<p_{-} \leqslant p(x) \leqslant p_{+} \leqslant+\infty\right\},
$$

Throughout the paper, we assume that $p \in C_{+}(X)$. we say that a real-valued continuous function $p($.$) is log-Hölder continuous in X$ if

$$
|p(x)-p(y)| \leq \frac{C}{-\log (d(x, y))}:(\forall x, y \in X) \text { such that } d(x, y) \leqslant \frac{1}{2},
$$

where $C$ is a constant.

For a $\mu$-measurable function $u: X \longrightarrow \mathbb{R}$ we define the modular

$$
\rho_{p(.)}(u)=\int_{X}|u(y)|^{p(y)} d \mu(y) .
$$

We define the variable exponent Lebesgue space on $X$ by

$$
L^{p(.)}(X)=\left\{u: X \longrightarrow \mathbb{R}, \quad \mu \text { - measurable }: \rho_{p(.)}\left(\frac{u}{\lambda}\right)<\infty, \text { for some } \lambda>0\right\} .
$$

Lemma 2.1. Under the norm $\|u\|_{p(.)}=\inf \left\{\lambda>0, \quad \rho_{p(.)}\left(\frac{u}{\lambda}\right) \leq 1\right\}$ the space $L^{p(.)}(X)$ is a Banach space.

Theorem 2.1. (i) Hölder inequality For any $u \in L^{p(.)}(X)$ and $v \in L^{p^{\prime}(.)}(X)$, we have

$$
\left|\int_{X} u v d x\right| \leq\left(\frac{1}{p^{-}}+\frac{1}{p^{\prime-}}\right)\|u\|_{p(.)}\|v\|_{p^{\prime}(.)} .
$$


(ii) $\|u\|_{p(.)}<1 \quad(\operatorname{resp},=1,>1) \quad \Longleftrightarrow \quad \rho_{p(.)}(u)<1 \quad(\operatorname{resp},=1,>1)$,

(iii) $\|u\|_{p(.)}>1 \Rightarrow\|u\|_{p(.)}^{p_{-}} \leq \rho_{p(.)}(u) \leq\|u\|_{p(.)}^{p_{+}}$and $\|u\|_{p(.)}<1 \Rightarrow\|u\|_{p(.)}^{p_{+}} \leq \rho_{p(.)}(u) \leq\|u\|_{p(.)}^{p_{-}}$

(iv) $\|u\|_{p(.)} \rightarrow 0 \Leftrightarrow \rho_{p(.)}(u) \rightarrow 0 \quad$ and $\quad\|u\|_{p(x)} \rightarrow \infty \Leftrightarrow \rho_{p(.)}(u) \rightarrow \infty$.

Theorem 2.2. Let $X$ be a locally compact doubling space and let $p^{+}<1$. The continuous functions with compact support are dense in $L^{p(.)}(X)$.

Proposition 2.1. If $1<p^{-}<p^{+}<\infty$ and $\mu$ is atomless, then $L^{p(.)}(X)$ is uniformly convex. From this it follows that $L^{p(.)}(X)$ is reflexive and has the Banach-Sacks propriety, namely, if $u_{i} \rightarrow u$ weakly in $L^{p(.)}(X)$ then $\frac{1}{i}\left(u_{1}+\ldots+u_{i}\right) \longrightarrow u$ strongly in $L^{p(.)}(X)$.

2.2. Weighted variable exponent Lebesgue spaces on metric measure spaces.[5]. Let $\omega$ be a function defined in $X, \omega$ is called a weight function in $X$ if it's $\mu$ - measurable and strictly positive $\mu$-almost every where in $X$.

For a $\mu$-measurable function $u: X \longrightarrow \mathbb{R}$ we define the modular

$$
\rho_{p(.), \omega}(u)=\int_{X} \omega(y)|u(y)|^{p(y)} d \mu(y) .
$$

We define the variable exponent Lebesgue space on $X$ by

$$
L^{p(.)}(X, \omega)=\left\{u: X \longrightarrow \mathbb{R}, \quad \mu \text { - measurable }: \rho_{p(.), \omega}\left(\frac{u}{\lambda}\right)<\infty, \text { for some } \lambda>0\right\}
$$

Lemma 2.2. If $\omega$ is a positive measurable function, then under the norm $\|u\|_{p(.), \omega}=\inf \left\{\lambda>0, \quad \rho_{p(.), \omega}\left(\frac{u}{\lambda}\right) \leq 1\right\}$ the space $L^{p(.)}(X, \omega)$ is a reflexive Banach space.

Theorem 2.3. (i) $\|u\|_{p(.), \omega}<1 \quad(\operatorname{resp},=1,>1) \quad \Longleftrightarrow \quad \rho_{p(.), \omega}(u)<1 \quad(\operatorname{resp},=1,>1)$,

(ii) $\|u\|_{p(.), \omega}>1 \Rightarrow\|u\|_{p(.), \omega}^{p_{-}} \leq \rho_{p(.), \omega}(u) \leq\|u\|_{p(.), \omega}^{p_{+}}$and $\|u\|_{p(.), \omega}<1 \Rightarrow\|u\|_{p(.), \omega}^{p_{+}} \leq \rho_{p(.), \omega}(u) \leq\|u\|_{p(.), \omega^{\prime}}^{p_{-}}$ (iii) $\|u\|_{p(.), \omega} \rightarrow 0 \Leftrightarrow \rho_{p(.), \omega}(u) \rightarrow 0$ and $\|u\|_{p(.), \omega} \rightarrow \infty \Leftrightarrow \rho_{p(.), \omega}(u) \rightarrow \infty$.

\section{Weighted variable exponent Sobolev spaces on metric measure spaces}

Let $(X, d)$ be a metric space with finite diameter, $\left(\operatorname{diam}(X)=\sup _{x, y \in X} d(x, y)<\infty\right)$ and equipped with a finite, positive Borel regular outer, atomless measure $\mu$. The $\operatorname{triplet}(X, d, \mu)$ will be fixed in the sequel and will be denoted by $X$.

$\omega$ is a positive measurable and finite function.

$p: X \longrightarrow[1,+\infty)$ is a variable exponent such that $1<p^{-}<p^{+}<\infty$.

Let $u: X \rightarrow[-\infty,+\infty]$ be a $\mu-$ measurable functions defined on $X$.

The average value of $u$ will be denoted by, $u_{X}=\frac{1}{\mu(X)} \int_{X} u d \mu=f_{X} u d \mu$.

We denote by $D(u)$ the set of all $\mu$-mesurable functions $g: X \rightarrow[0,+\infty]$, such that

$$
|u(x)-u(y)| \leqslant d(x, y)(g(x)+g(y))
$$

for every $x, y \in X \backslash F, x \neq y$, with $\mu(F)=0$. The set $F$ is called the exceptional set for $\mathrm{g}$, and $\mathrm{g}$ is called a Hajlasz gradient of $u$.

Note that the right-hand side of (3.1) is always defined for $x \neq y$. For the points $x, y \in X, x \neq y$ such that the left-hand side of (3.1) is undefined, we may assume that the left-hand side is $+\infty$. 
Definition 3.1. The Dirichlet-weighted variable exponent Sobolev spaces $L_{p(.), \mu}^{1}(X, \omega)$ is the space of all $\mu$-measurable functions $u$ such that $D(u) \cap L^{p(.)}(X, \omega) \neq \varnothing$. This space is equipped with the semi-norm

$$
\|u\|_{L_{p(.), u}^{1}(X, \omega)}=\inf \left\{\|g\|_{L^{p(.)}(X, \omega)} \quad: g \in D(u) \cap L^{p(.)}(X, \omega)\right\} .
$$

The weighted variable exponent Sobolev spaces on Arbitrary Metric space $X$ is

equipped with the norm

$$
M_{p(.), \mu}^{1}(X, \omega)=L^{p(.)}(X) \cap L_{p(.), \mu}^{1}(X, \omega),
$$

$$
\|u\|_{M_{p(.), \mu}^{1}(X, \omega)}=\|u\|_{L^{p(.)}(X)}+\|u\|_{L_{p(.), \mu}^{1}(X, \omega)} \cdot
$$

Lemma 3.1. If $(\forall y \in X) \omega(y) \geqslant 1$, then for every $u \in M_{p(.), \mu}^{1}(X, \omega)$ there is a unique $g \in D(u) \cap L^{p(.)}(X, \omega)$; denoted by $g_{u}$ such that

$$
\left\|g_{u}\right\|_{L^{p(.)}(X, \omega)}=\|u\|_{L_{p(.), \mu}^{1}(X, \omega)} .
$$

Proof. Let $\left(g_{i}\right) \subset D(u) \cap L^{p(.)}(X, \omega)$ a sequence such that

$$
\|u\|_{L_{p(.), u}^{1}(X, \omega)}=\lim _{i \rightarrow+\infty}\left\|g_{i}\right\|_{L^{p(.)}(X, \omega)}
$$

since $L^{p(.)}(X, \omega)$ is reflexive, there is a sub-sequence denoted again by $\left(g_{i}\right)$, and a function $g_{u} \in L^{p(.)}(X, \omega)$ such that $g_{i} \rightarrow g_{u}$ weakly in $L^{p(.)}(X, \omega)$. By Mazur lemma there exists a convex combinations $f_{k}=\sum_{i=k}^{m_{k}} \alpha_{k_{i}} g_{i}$, such that $f_{k} \longrightarrow g_{u}$ strongly in $L^{p(.)}(X, \omega)$, where $\alpha_{k_{i}} \geqslant 0$ and $\sum_{i=k}^{m_{k}} \alpha_{k_{i}}=1$.

Since every $g_{i} \in D(u) \cap L^{p(.)}(X, \omega)$, we have:

$$
\begin{aligned}
|u(x)-u(y)| & =\sum_{i=k}^{i=m_{k}} \alpha_{k_{i}}|u(x)-u(y)| \\
& \leqslant \sum_{i=k}^{i=m_{k}} \alpha_{k_{i}} d(x ; y)\left(g_{i}(x)+g_{i}(y)\right) \\
& =d(x ; y)\left(f_{k}(x)+f_{k}(y)\right), \quad \text { for } \mu-\text { almost all } x, y \in X
\end{aligned}
$$

Therefore $f_{k} \in D(u) \cap L^{p(.)}(X, \omega)$, for all $k$.

On the other hand, there exists a subsequence of $\left(f_{k}\right)$ witch converges to $g_{u}$ point wise $\mu$-almost everywhere in $X$, thus $g_{u} \in D(u) \cap L^{p(.)}(X, \omega)$.

Let $\varepsilon>0$, there is $k \in \mathbb{N}$ such that

$$
\left\|g_{i}\right\|_{L^{p(.)}(X, \omega)} \leqslant\|u\|_{L_{p(.), \mu}^{1}(X, \omega)}+\varepsilon, \quad \forall i \geqslant k .
$$

For this $k$ we have

$$
\left\|f_{k}\right\|_{L^{p(.)}(X, \omega)} \leqslant \sum_{i=k}^{m_{k}} \alpha_{k_{i}}\left\|g_{i}\right\|_{L^{p(.)}(X, \omega)} .
$$

Therefore

$$
\left\|f_{k}\right\|_{L^{p(.)}(X, \omega)} \leqslant\|u\|_{L_{p(.), \mu}^{1}(X, \omega)}+\varepsilon
$$

Thus

$$
\left\|g_{u}\right\|_{L^{p(.)}(X, \omega)}=\|u\|_{L_{p(.), \mu}^{1}(X, \omega)} \cdot
$$

Now suppose that there exists two minimal $g_{1}$ and $g_{2}$ with $g_{1} \neq g_{2}$, then

$$
\| g_{1}-\left.g_{2}\right|_{L^{p(.)}(X, \omega)}>0 .
$$


Let $G_{1}=\frac{g_{1}}{\left\|g_{1}\right\|_{L^{p(.)}(X, \omega)}}$ and $\quad G_{2}=\frac{g_{2}}{\left\|g_{2}\right\|_{L^{p(.)}(X, \omega)}}$. By the uniform convexity of the norm we have

$$
\left\|G_{1}+G_{2}\right\|_{L^{p(.)}(X, \omega)}<2
$$

then

$$
\left\|\frac{1}{2} g_{1}+\frac{1}{2} g_{2}\right\|_{L^{p(\cdot)}(X, \omega)}<\left\|g_{1}\right\|_{L^{p(\cdot)}(X, \omega)}
$$

witch is the contradiction since $\left(\frac{1}{2} g_{1}+\frac{1}{2} g_{2}\right) \in D(u) \cap L^{p(.)}(X, \omega)$.

With simple verification, we have the following lemma.

Lemma 3.2. Let $g_{1} \in D\left(u_{1}\right), g_{2} \in D\left(u_{2}\right)$ and $\alpha, \beta \in \mathbb{R}$. If $g \geqslant|\alpha| g_{1}+|\beta| g_{2}$, $\mu$.a.e. Then $g \in D\left(\alpha u_{1}+\beta u_{2}\right)$.

Lemma 3.3. Let $\left(u_{n}\right)$ and $\left(g_{n}\right)$ be two sequences of functions such that,

$$
\forall n \in \mathbb{N}: g_{n} \in D\left(u_{n}\right) .
$$

If $u_{n} \rightarrow u$ in $L^{p(.)}(X)$ and $g_{n} \rightarrow g$ in $L^{p(.)}(X, \omega)$, then $g \in D(u)$.

Proof. There are two subsequences of $\left(u_{n}\right)$ and $\left(g_{n}\right)$ which we denote again by $\left(u_{n}\right)$ and $\left(g_{n}\right)$ such that $u_{n} \rightarrow u$ and $g_{n} \rightarrow g$ u.a.e. For all $n \in \mathbb{N}$, let $F_{n}$ be the exceptional set for $g_{n}, \mathrm{H}$ a set of measure zero such that $u \rightarrow u$ and $g_{n} \rightarrow g$ on $H^{c}$, and $F=H \cup\left(\cup_{n} F_{n}\right)$. We have $\mu(F)=0$ and

$$
|u(x)-u(y)| \leqslant d(x, y)|g(x)+g(y)|, \forall x, y \in F .
$$

Therefore $g \in D(u)$.

Theorem 3.1. $\left(M_{p(\cdot), \mu}^{1}(X, \omega),\|\cdot\|_{M_{p(\cdot), \mu}^{1}(X, \omega)}\right)$ is a Banach space.

Proof. It is clear that $M_{p(.), \mu}^{1}(X, \omega)$ is a vector space.

Let $\left(u_{n}\right)_{n}$ be a Cauchy sequence in $M_{p(.), \mu}^{1}(X, \omega)$, then $\left(u_{n}\right)_{n}$ is a Cauchy sequence in $L^{p(.)}(X)$, thus there exists a function $u \in L^{p(.)}(X)$ such that

$u_{n} \rightarrow u$ in $L^{p(.)}(X)$. We can chose a subsequence denoted again $\left(u_{n}\right)_{n}$ such that $\left\|u_{n+1}-u_{n}\right\|_{M_{p(.), \mu}^{1}(X, \omega)}<2^{-n}$ and $u_{n} \rightarrow u \quad \mu-$ a.e in $X$. There exists a non-negative function $g_{n} \in L^{p(.)}(X, \omega)$ such that $\left\|g_{n}\right\|_{L^{p(.)}(X, \omega)}<2^{-n}$ and

$$
\left|\left(u_{n+1}-u_{n}\right)(x)-\left(u_{n+1}-u_{n}\right)(y)\right| \leqslant d(x, y)\left(g_{n}(x)+g_{n}(y)\right) \mu \text {-a.e in } X .
$$

By adding the last inequalities, we have for $n>m$,

$$
\left|\left(u_{n}-u_{m}\right)(x)-\left(u_{n}-u_{m}\right)(y)\right| \leqslant d(x, y)\left(\sum_{k=m}^{\infty} g_{k}(x)+\sum_{k=m}^{\infty} g_{k}(y)\right) \mu-\text { a.e in X. }
$$

Letting $n \rightarrow \infty$ yields

$$
\left|\left(u_{-} u_{m}\right)(x)-\left(u-u_{m}\right)(y)\right| \leqslant d(x, y)\left(\sum_{k=m}^{\infty} g_{k}(x)+\sum_{k=m}^{\infty} g_{k}(y)\right) \mu-\text { a.e in X. }
$$

Since

$$
\left\|\sum_{k=m}^{\infty} g_{k}\right\|_{L^{p(.)}(X, \omega)} \leqslant \sum_{k=m}^{\infty} 2^{-k}=2^{-m+1}
$$

the previous inequality implies that $\left(u_{-} u_{m}\right) \in M_{p(.), \mu}^{1}(X, \omega)$ and therefore $u \in M_{p(.), \mu}^{1}(X, \omega)$.

On the other hand, we have

$$
\left\|u-u_{m}\right\|_{L_{p(\cdot), \mu}^{1}(X, \omega)} \leqslant\left\|\sum_{k=m}^{\infty} g_{k}\right\| \leqslant 2^{-m+1},
$$

then $u_{m} \rightarrow u$ in $M_{p(.), \mu}^{1}(X, \omega)$. 
Theorem 3.2. $M_{p(.), \mu}^{1}(X, \omega)$ is reflexive.

Proof. By proposition2.1 We have $L^{p(.)}(X)$ is a reflexive Banach space and by theorem3.1 $M_{p(.), \mu}^{1}(X, \omega)$ is closed, thus $M_{p(.), \mu}^{1}(X, \omega)$ is reflexive.

Lemma 3.4. The function $u$ belongs to $M_{p(.), \mu}^{1}(X, \omega)$ if an only if $u \in L^{p(.)}(X)$ and there exists two sequences $\left(u_{n}\right) \subset$ $L^{p(.)}(X)$ and $\left(g_{n}\right) \subset D\left(u_{n}\right) \cap L^{p(.)}(X, \omega)$, such that $u_{n} \rightarrow u \mu$-a.e and $g_{n} \rightarrow g$-a.e for some $g \in L^{p(.)}(X, \omega)$.

Proof. The same as in [11] lemma 2.5.

Lemma 3.5. Let $u_{1}, u_{1} \in M_{p(.), \mu}^{1}(X, \omega)$. If $g_{1} \in D\left(u_{1}\right)$ and $g_{2} \in D\left(u_{2}\right)$, then :

$$
\begin{aligned}
& \text { (i) } u=\max \left(u_{1}, u_{2}\right) \in M_{p(.), \mu}^{1}(X, \omega) \text { and } g=\max \left(g_{1}, g_{2}\right) \in D(u) \cap L^{p(.)}(X, \omega) \text {. } \\
& \text { (ii) } v=\min \left(u_{1}, u_{2}\right) \in M_{p(.), \mu}^{1}(X, \omega) \text { and } h=\max \left(g_{1}, g_{2}\right) \in D(v) \cap L^{p(.)}(X, \omega) \text {. }
\end{aligned}
$$

Proof. We only prove the case (i), the proof of (ii) is similar.

Let $F_{1}$ and $F_{2}$ the exceptional sets for $g_{1}$ and $g_{2}$, respectively. Clearly $u \in L^{p(.)}(X)$ and $g \in L^{p(.)}(X, \omega)$. It remains to prove that $g \in D(u)$. Let $G=\left\{x \in X \backslash\left(F_{1} \cup F_{2}\right): u_{1}(x) \geqslant u_{2}(x)\right\}$.

If $x, y \in G$, then

$$
|u(x)-u(y)|=\left|u_{1}(x)-u_{1}(y)\right| \leqslant d(x, y)\left(g_{1}(x)+g_{1}(y)\right) .
$$

Analogously, for $x, y \in X \backslash G$, we obtain $|u(x)-u(y)| \leqslant d(x, y)\left(g_{2}(x)+g_{2}(y)\right)$.

For the remaining cases, let $x \in G$ and $y \in X \backslash G$ :

If $u_{1}(x) \geqslant u_{2}(y)$, then

$$
|u(x)-u(y)|=u_{1}(x)-u_{2}(y) \leqslant u_{1}(x)-u_{1}(y) \leqslant d(x, y)\left(g_{1}(x)+g_{1}(y)\right) .
$$

If $u_{1}(x)<u_{2}(y)$, then

$$
|u(x)-u(y)|=u_{2}(y)-u_{1}(x) \leqslant u_{2}(y)-u_{2}(x) \leqslant d(x, y)\left(g_{2}(x)+g_{2}(y)\right) .
$$

The case $x \in X \backslash G$ and $y \in G$ follows by symmetry and hence

$$
|u(x)-u(y)| \leqslant d(x, y)(g(x)+g(y))
$$

for all $x, y \in X \backslash\left(F_{1} \cup F_{2}\right)$, with $\mu\left(\left(F_{1} \cup F_{2}\right)=0\right.$.

Theorem 3.3. (The Poincare inequality).

If $p($.$) is log-Hölder continuous, then exists C>0$, depending only of $p^{-}, p^{+}$and $\mu(X)$ such that

$$
\left(\forall u \in M_{p(.), \mu}^{1}(X, \omega)\right)\left(\forall g \in D(u) \cap L^{p(.)}(X, \omega)\right):\left\|u-u_{X}\right\|_{p(.), \omega} \leqslant C \operatorname{diam}(X)\|g\|_{p(.), \omega} .
$$

Proof. Integrating the inequality $|u(x)-u(y)| \leqslant d(x, y)(g(x)+g(y))$ over $y$ we obtain

Thus,

$$
\left|u(x)-f_{X} u(y) d \mu(y)\right| \leqslant f_{X}|u(x)-u(y)| d \mu(y) \leqslant \operatorname{diam}(X)\left(g(x)+f_{X} g(y) d \mu(y)\right) .
$$

Therefore

$$
\left|u(x)-u_{X}\right| \leqslant \operatorname{diam}(X)\left(g(x)+\frac{C^{\prime}\|1\|_{p^{\prime}(.), \omega}}{\mu(X)}\|g\|_{p(.), \omega}\right)
$$

where $C=C\left(p^{-}, p^{-}, \mu(X)\right)$.

$$
\left\|u-u_{X}\right\|_{p(.), \omega)} \leqslant C \operatorname{diam}(X)\|g\|_{L^{p(.)}(X, \omega)^{\prime}}
$$

Theorem 3.4. Let $p$ (.) a log-Hölder continuous function. If $(\forall y \in X) \omega(y) \geqslant 1$, and $\omega \in L^{p^{\prime}(.)}(X)$ then for every $u \in M_{p(.), \mu}^{1}(X, \omega)$ and every $\varepsilon>0$ there exists a Lipschitz function $h \in M_{p(.), \mu}^{1}(X, \omega)$ such that 
(i) $\mu(\{x \in X: u(x) \neq h(x)\}) \leqslant \varepsilon$

(i) $\quad\|u-h\|_{M_{p(.), \mu}^{1}(X, \omega)} \leqslant \varepsilon$.

Proof. We fix $u \in M_{p(.), \mu}^{1}(X, \omega)$ and denote by $g \in L^{p(.)}(X, \omega)$ a Hajlasz gradient of $u$. We write $E_{n}=\{x \in X$ : $|u(x)| \leqslant n$ and $g(x) \leqslant n\}$.

We have

$$
\forall y \in E_{n}^{C}: n \leqslant|u(y)|+|g(y)| .
$$

Thus

therefore

$$
\mu\left(E_{n}^{c}\right) \leqslant \frac{2^{p^{+}-1}}{n^{p^{+}}} \int_{E_{n}^{C}}\left(|u(y)|^{p(y)}+|g(y)|^{p(y)}\right) d \mu(y)
$$

$$
\mu\left(E_{n}^{c}\right) \leqslant \frac{2^{p^{+}-1}}{n^{p^{+}}}\left(\int_{E_{n}^{C}}|u(y)|^{p(y)} d \mu(y)+\int_{E_{n}^{C}} \omega(y)|g(y)|^{p(y)}\right) .
$$

Thus

$$
\mu\left(E_{n}^{c}\right) \leqslant \frac{2^{p^{+}-1}}{n^{p^{+}}}\left(\rho_{p(.)}(u)+\rho_{p(.), \omega}(g)\right)
$$

then

$$
\lim _{n \rightarrow+\infty} \mu\left(E_{n}^{c}\right)=0 .
$$

The restriction $u \backslash E_{n}$ is Lipschitz with the constant $2 n$. We can extend $u \backslash E_{n}$ by Mcshane extention [12], to all of X as a Lipschitz function by defining

$$
\bar{u}(x)=\inf _{y \in E_{n}}\left\{u \backslash E_{n}(y)+2 n \operatorname{dist}(x, y)\right\} .
$$

We put:

$$
u_{n}=(\operatorname{sing} \bar{u}) \min (|\bar{u}|, n) .
$$

It is clear that $u_{n}$ is Lipschitz with constant $2 n, u \backslash E_{n}=u_{n} \backslash E_{n},\left|u_{n}\right| \leqslant n$ and for all $n \geqslant 1$

$$
\mu\left(\left\{x: u(x) \neq u_{n}(x)\right)\right\} \leqslant \mu\left(E_{n}^{c}\right) \rightarrow 0 \text { as } n \rightarrow \infty .
$$

On the other hand, we have

$\rho_{p(.)}\left(u_{n}-u\right)=\int_{X}\left|u_{n}(y)-u(y)\right|^{p(y)} d \mu(y)=\int_{E_{n}^{c}}\left|u_{n}(y)-u(y)\right|^{p(y)} d \mu(y)$.

Thus

We have

$$
\rho_{p(.)}\left(u_{n}-u\right) \leqslant 2^{p^{+}-1}\left(\int_{E_{n}^{c}}\left|u_{n}(y)\right|^{p(y)} d \mu(y)+\int_{E_{n}^{c}}|u(y)|^{p(y)} d \mu(y)\right) .
$$

$$
\int_{E_{n}^{c}}\left|u_{n}(y)\right|^{p(y)} d \mu(y) \leqslant \int_{E_{n}^{c}} n^{p(y)} d \mu(y) \leqslant 2^{p^{+}-1} \int_{E_{n}^{c}}\left(|u(y)|^{p(y)}+|g(y)|^{p(y)}\right) d \mu(y) .
$$

Therefore

$$
\rho_{p(.)}\left(u_{n}-u\right) \leqslant 2^{p^{+}-1}\left(2^{p^{+}-1} \int_{E_{n}^{c}}\left(|u(y)|^{p(y)}+\omega(y)|g(y)|^{p(y)}\right) d \mu(y)+\int_{E_{n}^{c}}|u(y)|^{p(y)} d \mu(y)\right) .
$$

Since $\lim _{n \rightarrow+\infty} \mu\left(E_{n}^{c}\right)=0$, then

$$
\lim _{n \rightarrow+\infty} \rho_{p(.)}\left(u_{n}-u\right)=0
$$

Thus

It remains to estimate the gradient, let

$$
\lim _{n \rightarrow+\infty}\left\|u_{n}-u\right\|_{L^{p(.)}(X)}=0 .
$$

$$
g_{n}=\left\{\begin{array}{ccc}
0 & \text { for } & x \in E_{n} \\
g(x)+3 n & \text { for } & x \in E_{n}^{c}
\end{array}\right.
$$


It is easy to check that

$$
\left|\left(u-u_{n}\right)(x)-\left(u-u_{n}\right)(y)\right| \leqslant d(x, y)\left(g_{n}(x)+g_{n}(y)\right), \text { for } \mu-\text { almost all } x, y \in X .
$$

We have

$$
\int_{X} \omega(y)\left|g_{n}(y)\right|^{p(y)} d \mu(y)=\int_{E_{n}^{c}} \omega(y)\left|g_{n}(y)\right|^{p(y)} d \mu(y) .
$$

Thus

We have

$$
\int_{X} \omega(y)\left|g_{n}(y)\right|^{p(y)} d \mu(y) \leqslant 2^{p^{+}-1}\left(\int_{E_{n}^{c}} \omega(y)|g(y)|^{p(y)} d \mu(y)+\int_{E_{n}^{c}} \omega(y)(3 n)^{p(y)} d \mu(y)\right) .
$$

On the other hand we have

$$
\int_{E_{n}^{c}} \omega(y)(3 n)^{p(y)} d \mu(y) \leqslant\left(\frac{1}{p^{-}}+\frac{1}{p^{\prime-}}\right)(3 n)^{p+} \mu\left(E_{n}^{c}\right)\|\omega\|_{L^{p^{\prime}(.)}(X)} .
$$

$$
\mu\left(E_{n}^{c}\right) \leqslant \int_{E_{n}^{c}}\left(\frac{|u(y)|+|g(y)|}{n}\right)^{p(y)} d \mu(y),
$$

thus

$$
n^{p^{+}} \mu\left(E_{n}^{c}\right) \leqslant \int_{E_{n}^{c}}(|u(y)|+|g(y)|)^{p(y)} d \mu(y) .
$$

Hence

$$
n^{p^{+}} \mu\left(E_{n}^{c}\right) \leqslant 2^{p^{+}-1}\left(\int_{E_{n}^{c}}|u(y)|^{p(y)} d \mu(y)+\int_{E_{n}^{c}} \omega(y)|g(y)|^{p(y)} d \mu(y)\right),
$$

which implies

$$
n^{p^{+}} \mu\left(E_{n}^{c}\right) \longrightarrow 0 \text { as } n \longrightarrow \infty \text {. }
$$

Then

$$
\int_{E_{n}^{c}} \omega(y)|g(y)|^{p(y)} d \mu(y) \longrightarrow 0, \text { as } n \longrightarrow \infty .
$$

Thus $\left\|g_{n}\right\|_{L^{p(.)}(X, \omega)} \rightarrow 0$, as $n \rightarrow \infty$. Therefore

$$
\lim _{n \rightarrow+\infty}\left\|u_{n}-u\right\|_{M_{p(.), \mu}^{1}(X, \omega)}=0 .
$$

\section{Capacity on Musielac- Orlic-Sobolev space on metric space}

Definition 4.1. let $T$ the classe of Borel sets in $X$, and a function $C: T \rightarrow[0,+\infty]$.

1) $C$ is called capacity if the following axioms are satisfied:

i) $C(\varnothing)=0$

ii) $E \subset F \Rightarrow C(E) \leqslant C(F)$, for all $E$ and $F$ in $T$.

iii) for all sequences $\left(E_{n}\right) \subset T$ :

$$
C\left(\bigcup_{n} E_{n}\right) \leqslant \sum_{n} C\left(E_{n}\right)
$$

2) $C$ is called outer capacity if for all $E \in T$ :

$$
C(E)=\inf \{C(O): O \supset X, O \text { open }\}
$$

3)A property that holds true except perhaps on a set of capacity zero is said to be true C-quasieverywhere, (abbreviated C-q.e).

4) $f$ and $\left(f_{n}\right)$ are real-valued function finite $C$-q.e. We say that $\left(f_{n}\right)$ converges to $f$ in $C$-capacity if:

$$
\forall \varepsilon>0, \lim _{n \rightarrow+\infty} C\left(\left\{x:\left|f_{n}(x)-f(x)\right|>\varepsilon\right\}\right)=0 .
$$


5) $f$ and $\left(f_{n}\right)$ are real-valued function finite C-q.e. We say that $\left(f_{n}\right)$ converges to $f$ C-quasi uniformly, (abbreviated C-q.u) if:

$$
\forall \varepsilon>0, \exists X \in T: C(X)<\varepsilon \text { and }\left(f_{n}\right) \text { converges to } f \text { uniformly, on } X^{c}
$$

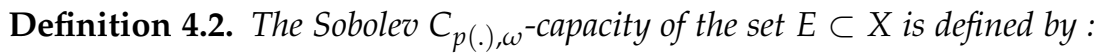

$$
C_{p(.), \omega}(E)=\inf _{u \in A_{p(.), \omega}(E)}\|u\|_{M_{p(\cdot), \mu}^{1}(X, \omega)}
$$

Where

$$
A_{p(.), \omega}(E)=\left\{u \in M_{p(.), \mu}^{1}(X, \omega): u \geqslant 1 \text { on an open set containing } E \text { and } u \geqslant 0\right\} .
$$

If $A_{p(.), \omega}(E)=\varnothing$ we set $C_{p(.), \omega}(E)=\infty$. Functions belonging to $A_{p(.), \omega}(E)$ are called admissible functions for $E$.

Remark 4.1. In the defition of $C_{p(.), \omega}(E)$, we can restrict ourselves to those admissible functions $u$ for which $0 \leqslant u \leqslant 1$.

Proof. if $A_{p(.), \omega}^{\prime}(E)=\left\{u \in A_{p(.), \omega}(E): 0 \leqslant u \leqslant 1\right\}$, then $A_{p(.), \omega}^{\prime}(E) \subset A_{p(.), \omega}(E)$ implies

$$
C_{p(.), \omega}(E) \leqslant \inf _{u \in A_{p(.), \omega}^{\prime}(E)}\|u\|_{M_{p(.), \mu}^{1}(X, \omega)} .
$$

For the reverse inequality, let $\varepsilon>0$ and take $u \in A_{p(.), \omega}$ such that

$$
\|u\|_{M_{p(\cdot), \mu}^{1}(X, \omega)}<C_{p(.), \omega}(E)+\varepsilon .
$$

Then by lemma 3.5, we have $v=\max (0, \min (u, 1))$ belongs to $A_{p(.), \omega}^{\prime}(E)$. Thus

$$
\inf _{f \in A_{p(.), \omega}^{\prime}(E)}\|f\|_{M_{p(\cdot), \mu}^{1}(X, \omega)} \leqslant\|v\|_{M_{p(.), \mu}^{1}(X, \omega)} \leqslant C_{p(.), \omega}(E)+\varepsilon .
$$

Letting $\varepsilon \longrightarrow 0$, we obtain

This complete the proof.

$$
\inf _{f \in A_{p(.), \omega}^{\prime}}\|f\|_{M_{p(.), \mu}^{1}(X, \omega)} \leqslant C_{p(.), \omega}(E)
$$

Theorem 4.1. $C_{p(.), \omega}$ is an outer capacity.

Proof. It is obvious that $C_{p(.), \omega}(\varnothing)=0$ and $C_{p(.), \omega}(E) \leqslant C_{p(.), \omega}(F)$ if $E \subset F$.

Let $\left(E_{i}\right) \subset X$, so that $\sum_{i} C_{p(.), \omega}\left(E_{i}\right)<+\infty$, then $C_{p(.), \omega}\left(E_{i}\right)<+\infty ; \forall i \in \mathbb{N}$.

Therefore $\forall \varepsilon>0, \exists u_{i} \in A_{p(.), \omega}\left(E_{i}\right)$ and $g_{u_{i}} \in D\left(u_{i}\right) \cap L_{\varphi}(X)$ so that

$$
\left\|u_{i}\right\|_{L^{p(.)}(X)}+\left\|g_{u_{i}}\right\|_{L^{p(.)}(X, \omega)} \leqslant C_{p(.), \omega}\left(E_{i}\right)+\varepsilon .2^{-i} .
$$

We show that $v=\sup _{i} u_{i}$ is an admissible function for $\bigcup_{i=1}^{\infty} E_{i}$ and $g=\sup _{i} g_{u_{i}} \in D(v) \cap L^{p(.)}(X, \omega)$. Let $v_{k}=\max _{1 \leqslant i \leqslant k} u_{i}$, by lemma 3.5 the function $g_{v_{k}}=\max _{1 \leqslant i \leqslant k} g_{u_{i}}$ belongs to $D\left(v_{k}\right) \cap L^{p(.)}(X, \omega)$. Since $v_{k} \rightarrow v$ u.a.e and $g_{v_{k}} \rightarrow g$ p.a.e, lemma 3.4 gives $v \in M_{p(.), \mu}^{1}(X, \omega)$.

Clearly $v \geqslant 1$ in a neighbourhood of $\bigcup_{i=1}^{\infty} E_{i}$. Then

$$
C_{p(.), \omega}\left(\bigcup_{i=1}^{\infty} E_{i}\right) \leqslant\|v\|_{M_{p(.), \mu}^{1}(X, \omega)} \leqslant \sum_{i=1}^{\infty}\left(\left\|u_{i}\right\|_{L^{p(.)}(X)}+\left\|g_{u_{i}}\right\|_{L^{p(.)}(X, \omega)}\right) \leqslant \sum_{i=1}^{\infty} C_{p(.), \omega}\left(E_{i}\right)+\varepsilon .
$$

The claim follows by letting $\varepsilon \rightarrow 0$. Hence $C_{p(.), \omega}$ is a capacity.

It remains to prove that $C_{p(.), \omega}$ is outer.

Indeed, $C_{p(.), \omega}(E) \leqslant \inf \left\{C_{p(.), \omega}(O): E \subset O, O\right.$ is open $\}$. For the reverse inequality, let $\varepsilon>0$ and $u \in A_{p(.), \omega}(E)$ such that $\|u\|_{M_{p(.), \mu}^{1}(X, \omega)} \leqslant C_{p(.), \omega}(E)+\varepsilon$. Since $u \in A_{p(.), \omega}(E)$, there is an open set $\mathrm{O}, E \subset O$, such that $u \geqslant 1$ on $\mathrm{O}$. 
Then $C_{p(.), \omega}(O) \leqslant\|u\|_{M_{p(.), \mu}^{1}(X, \omega)} \leqslant C_{p(.), \omega}(E)+\varepsilon$.

The claim follows by letting $\varepsilon \rightarrow 0$.

Theorem 4.2. If $\left(K_{n}\right)$ is a decreasing sequence of compacts and $K=\bigcap_{n} K_{n}$, then

$$
\lim _{n \rightarrow+\infty} C_{p(.), \omega}\left(K_{n}\right)=C_{p(.), \omega}(K) .
$$

Proof. First, we observe that $\lim _{n \rightarrow+\infty} C_{p(.), \omega}\left(K_{n}\right) \geqslant C_{p(.), \omega}(K)$.

Let $O$ be an open set containing $K$. By the compactness of $K, K_{i} \subset O$ for all sufficiently large $i$. Thus $\lim _{n \rightarrow+\infty} C_{p(.), \omega}\left(K_{n}\right) \leqslant C_{p(.), \omega}(O)$ and since $C_{p(.), \omega}$ is an outer capacity, we obtain the claim by taking infimum over all open set $\mathrm{O}$ containing $\mathrm{K}$.

Theorem 4.3. Let $E \subset X$. If there exists $f \in M_{p(.), \mu}^{1}(X, \omega)$ such that $f=+\infty$ on an open set containing $E$ then, $C_{p(.), \omega}(E)=0$.

Proof. If there exists $f \in M_{p(.), \mu}^{1}(X, \omega)$ such that $f=+\infty$ on an open set $O$ containing $E$, then $f \geqslant \alpha$ on $O$ for all $\alpha>0$. Therefore,

Letting $\alpha \longrightarrow+\infty$, we obtain $C_{p(.), \omega}(E)=0$.

$$
\forall \alpha>0 \quad: C_{p(.), \omega}(E) \leqslant \frac{1}{\alpha}\|f\|_{M_{p(.), \mu}^{1}(X, \omega)} .
$$

Theorem 4.4. Let's consider the following propositions:

i) $f_{n} \longrightarrow f$ in $M_{p(.), \mu}^{1}(X, \omega)$ strongly.

ii) $f_{n} \longrightarrow f$ in $C_{p(.), \omega}$ - capacity.

iii) There is a subsequence $\left(f_{n_{j}}\right)$ such that $: f_{n_{j}} \longrightarrow f, C_{p(.), \omega}-q . u$.

iv) $f_{n_{j}} \longrightarrow f, \quad C_{p(.), \omega}-$ q.e.

We have i) $\Rightarrow$ ii $\Rightarrow$ iii $) \Rightarrow i v)$.

Proof. Let show that $i) \Rightarrow i i)$.

By theorem 4.3 , we have $f$ and $f_{n}$ are finites for every $\mathrm{n} ; C_{p(.), \omega}-$ q.e.

Let $\varepsilon>0$; then

$$
C_{p(.), \omega}\left(\left\{x:\left|f_{n}-f\right|(x)>\varepsilon\right\}\right) \leqslant \frac{\left\|f-f_{n}\right\|_{M_{p(.), \mu}^{1}(X, \omega)}}{\varepsilon} .
$$

Let show that $i i) \Rightarrow$ iii).

Let $\varepsilon>0 \exists f_{n_{j}}$ such that

$$
C_{p(.), \omega}\left(\left\{x:\left|f_{n_{j}}-f\right|(x)>2^{-j}\right\}\right)<\varepsilon .2^{-j}
$$

We put

$$
E_{j}=\left\{x:\left|f_{n_{j}}-f\right|(x)>2^{-j}\right\} \quad \text { and } G_{m}=\bigcup_{j \geqslant m} E_{j} .
$$

We have $C_{p(.), \omega}\left(G_{m}\right) \leqslant \sum_{j \geqslant m} \varepsilon .2^{-j}<\varepsilon$.

On the other hand :

$$
\forall x \in\left(G_{m}\right)^{c}, \forall j \geqslant m:\left|f_{n_{j}}-f\right|(x) \leqslant 2^{-j} .
$$

Thus $f_{n_{j}} \longrightarrow f C_{p(.), \omega}-q . u$.

Let show that iii) $\Rightarrow$ iv).

We have $\forall j \in \mathbb{N}, \exists E_{j} \subset X: C_{p(.), \omega}\left(E_{j}\right) \leqslant \frac{1}{j}$ and $f_{n_{j}} \longrightarrow f$ on $\left(E_{j}\right)^{c}$.

We put $E=\bigcap_{j} X_{j}$, then $C_{p(.), \omega}(E)=0$ and $f_{n_{j}} \longrightarrow f$ on $E^{c}$. 
Theorem 4.5. If $f_{n}, f \in M_{p(.), \mu}^{1}(X, \omega)$ such that $f_{n} \rightarrow f$ weakly in $M_{p(.), \mu}^{1}(X, \omega)$, then:

$$
\liminf f_{n} \leqslant f \leqslant \limsup f_{n}, C_{p(.), \omega}-\text { q.e. }
$$

Proof. By theorem3.2 $M_{p(.), \mu}^{1}(X, \omega)$ is reflexive. By the Banach-Saks theorem, there is a subsequence denoted again $\left(f_{n}\right)$ such that the sequence $g_{n}=\frac{1}{n} \sum_{i=1}^{n} f_{i}$ converges to $f$ strongly in $M_{p(.), \mu}^{1}(X, \omega)$. By theorem 4.4, there is a sub-sequence of $\left(g_{n}\right)$ denoted again $\left(g_{n}\right)$ such that

$$
\lim _{n \rightarrow+\infty} g_{n}=f C_{p(.), \omega}-q . e .
$$

On the other hand,

$$
\liminf f_{n} \leqslant \lim _{n \rightarrow+\infty} g_{n}
$$

Therefore,

$$
\liminf \leqslant f \quad C_{p(.), \omega}-\text { q.e. }
$$

For the second inequality, it suffices to replace $f_{n}$ by $\left(-f_{n}\right)$ in the first inequality.

Theorem 4.6. Assuming that $\omega$ is a positive measurable and finite function. Let $\left(O_{n}\right)$ an increasing sequence of open subsets of $X$ and $O=\bigcup_{n} O_{n}$, then

$$
\lim _{n \rightarrow+\infty} C_{p(.), \omega}\left(O_{n}\right)=C_{p(.), \omega}(O) .
$$

Proof. We have $\lim _{n \rightarrow+\infty} C_{p(.), \omega}\left(O_{n}\right) \leqslant C_{p(.), \omega}(O)$. For the reverse Inequality, if $\lim _{n \rightarrow+\infty} C_{p(.), \omega}\left(O_{n}\right)=+\infty$ there is nothing to show.

Assuming that $\lim _{n \rightarrow+\infty} C_{p(.), \omega}\left(O_{n}\right)<+\infty$, we have

$$
\forall n \in \mathbb{N}, \quad \exists f_{n} \in M_{p(.), \mu}^{1}(X, \omega): f_{n} \geqslant 1 \text { on } O_{n} \text { and }\left\|f_{n}\right\|_{M_{p(.), \mu}^{1}(X, \omega)} \leqslant C_{p(.), \omega}\left(O_{n}\right)+\frac{1}{n} .
$$

Then

$$
\left\|f_{n}\right\|_{L^{p(\cdot)}(X)}+\left\|g_{n}\right\|_{L^{p(.)}(X, \omega)} \leqslant C_{p(\cdot), \omega}\left(O_{n}\right)+\frac{1}{n} .
$$

Where $g_{n} \in D\left(f_{n}\right) \cap L^{p(.)}(X, \omega)$. Now $\left(f_{n}\right)$ and $\left(g_{n}\right)$ are two bounded sequences in $L^{p(.)}(X)$ and $L^{p(.)}(X, \omega)$ respectively, hence there exists two sub-sequences, which we denote again by $\left(f_{n}\right)$ and $\left(g_{n}\right)$, such that $f_{n} \rightarrow f$ weakly in $L^{p(.)}(X)$ and $g_{n} \rightarrow g$, weakly in $L^{p(.)}(X, \omega)$. Thus

$$
\|f\|_{L^{p(\cdot)}(X)} \leqslant \liminf _{n}\left\|f_{n}\right\|_{L^{p(\cdot)}(X)} \text { and }\|g\|_{L^{p(.)}(X, \omega)} \leqslant \liminf _{n}\left\|g_{n}\right\|_{L^{p(.)}(X, \omega)} .
$$

Hence

On the other hand we have

$$
\|f\|_{M_{p(.), \mu}^{1}(X, \omega)} \leqslant \lim _{n \rightarrow+\infty} C_{p(.), \omega}\left(O_{n}\right) .
$$

$$
\forall n \in \mathbb{N}: f_{n} \geqslant 1 \text { on } O_{n}
$$

thus, by theorem 4.5, $f \geqslant 1$ on $O C_{p(.), \omega}-$ q.e.

Let $Y$ be a subset of $O$ where $f \geqslant 1$, then $C_{p(.), \omega}(O)=C_{p(.), \omega}(Y)$.

Thus,

$$
C_{p(.), \omega}(O) \leqslant \lim _{n}\left(C_{p(.), \omega}\left(O_{n}\right)\right)
$$

Theorem 4.7. We have

$$
(\exists \alpha>0)(\forall E \subset X), \mu(E) \leqslant \alpha \cdot C_{p(.), \omega}(E) .
$$

Proof. Let $E \subset X$ and $f \in A_{p(.), \omega}(E)$, then $f \geqslant 1$ on an open set $O$ containing $E$ and $f \geqslant 0$. We have

$$
\mu(E) \leqslant \mu(O) \leqslant \int_{O} f(x) d \mu(x) \leqslant \int_{X} f(x) d \mu(x) .
$$


By Holder inequality we have

Therefore

$$
\int_{X} f(x) d \mu(x) \leqslant 2\|f\|_{L^{p(.)}(X)}\left\|\mathbf{1}_{X}\right\|_{L^{p^{\prime}(.)(X)}} \cdot
$$

where

$$
\mu(E) \leqslant 2(\mu(X))^{\gamma} \quad\|f\|_{M_{p(\cdot), \mu}^{1}(X, \omega)^{\prime}}
$$

$$
\gamma= \begin{cases}p^{\prime-}, & \text { if }\left\|\mathbf{1}_{X}\right\|_{L^{\left.p^{(} .\right)(X)}} \geqslant 1, \\ p^{\prime+}, & \text { if }\left\|\mathbf{1}_{X}\right\|_{L^{p^{\prime}(.)(X)}}<1 .\end{cases}
$$

We obtain the claim by passing to inf on $f \in A_{p(.), \omega}$.

Corollary 4.1. If $\left(f_{n}\right)_{n}$ is a sequence which converges to $f$ in $M_{p(.), \mu}^{1}(X, \omega)$, then there exists a subsequence of $\left(f_{n}\right)_{n}$ which converges to $f$, $\mu$-a.e.

Proof. It is an immediate consequence of theorem 4.4 and theorem 4.7.

Theorem 4.8. For each $f \in M_{p(.), \mu}^{1}(X, \omega)$, there is a $C_{p(.), \omega}$-quasi continuous function $g \in M_{p(.), \mu}^{1}(X, \omega)$ such that $f=g$, p.a.e.

Proof. Let $f \in M_{p(.), \mu}^{1}(X, \omega)$. By theorem 3.4, there exists a sequence $\left(f_{n}\right)$ of lipschitz function, such that $f_{n} \longrightarrow f$ in $M_{p(.), \mu}^{1}(X, \omega)$.

By theorem 4.4 , there exists a subsequence of $\left(f_{n}\right)$ denoted again by $\left(f_{n}\right)$ such that $f_{n} \longrightarrow f C_{p(.), \omega}-q . u$. The claim follows by theorem 4.7.

Theorem 4.9. We have the following results of the $C_{p(.), \omega}$ capacity:

1) If $O$ is an open set of $X$ and $E \subset X$ such that $\mu(E)=0$, then

$$
C_{p(.), \omega}(O)=C_{p(.), \omega}(O-E) .
$$

2) Let $u$ and $v$ are $C_{p(.), \omega}$-quasicontinuous functions in $X$, we have

i) if $u=v$, almost everywhere in an open set $O \subset X$, then

$$
u=v \quad C_{p(.), \omega} \text {-quasieverywhere in } O,
$$

ii) if $u \leqslant v$, almost everywhere in an open set $O \subset X$, then

$$
u \leqslant v \quad C_{p(.), \omega} \text { - quasieverywhere in } O .
$$

Proof. 1) It obvious that $C_{p(.), \omega}(O) \geqslant C_{p(.), \omega}(O-E)$. Let $u \in A_{p(.), \omega}(O-E)$ thus $u \geqslant 1$ in an open set containing $O-E$. Let the function $f$ define as

$$
\left\{\begin{array}{cl}
f(x)=u(x) & , \text { if } x \in X-E \\
f(x)=1 & \text {, if } x \in E .
\end{array}\right.
$$

We have $f \in A_{p(.), \omega}(O)$ and $\|f\|_{M_{p(\cdot), \mu}^{1}(X, \omega)}=\|u\|_{M_{p(\cdot), \mu}^{1}(X, \omega)}$, thus

$$
C_{p(.), \omega}(O) \leqslant\|u\|_{M_{p(\cdot), \mu}^{1}(X, \omega)}
$$

and by passing to inf we get $C_{p(.), \omega}(O) \leqslant C_{p(.), \omega}(O-E)$.

2) Since $C_{p(.), \omega}$ is an outer capacity we get the results by [?].

\section{Weighted variable exponents Sobolev spaces with zero boundary values}

Let $(X, d)$ be a metric space with finite diameter, $\left(\operatorname{diam}(X)=\sup _{x, y \in X} d(x, y)<\infty\right)$ and equipped with a finite, positive Borel regular outer, atomless measure $\mu$. The triplet $(X, d, \mu)$ will be fixed in the sequel and will be denoted 
by $X$.

$\omega$ is a positive measurable and finite function.

$p: X \longrightarrow[1,+\infty)$ is a variable exponent such that $1<p^{-}<p^{+}<\infty$.

Definition 5.1. Let $E$ be a subset of $X$, we say that a function $u$ belongs to the weighted variable exponents Sobolev spaces with zero boundary values, and denoted $u \in B_{p(.)}^{1,0}(E, \omega)$, if there is a $C_{p(.), \omega}$-quasicontinuous functions $\tilde{u} \in M_{p(.), \mu}^{1}(X, \omega)$ such that $\tilde{u}=u$ almost everywhere in $E$ and $\tilde{u}=0 C_{p(.), \omega}$-quasi-everywhere in $X \backslash E$.

The space $B_{p(.)}^{1,0}(E, \omega)$ is endowed with the norm

$$
\|u\|_{B_{p(.)}^{1,0}(E, \omega)}=\|\tilde{u}\|_{M_{p(\cdot), \mu}^{1}(X, \omega)} .
$$

Remark 5.1. The norm of $B_{p(.)}^{1,0}(E, \omega)$ does not depend on the choice of the $C_{p(.), \omega}$-quasicontinuous representative since $C_{p(.), \omega}(D)=0$ implies that $\mu(D)=0$ for every $D \subset X$.

Theorem 5.1. $B_{p(.)}^{1,0}(E, \omega)$ is a Banach spaces.

Proof. Let $\left(u_{i}\right)_{i}$ be a Cauchy sequence in $B_{p(.)}^{1,0}(E, \omega)$, for every $i$ there is a $C_{p(.), \omega}$-quasicontinuous function $\tilde{u}_{i} \in M_{p(.), \mu}^{1}(X, \omega)$ such that $\tilde{u}_{i}=u_{i}$ almost everywhere in $E$ and $\tilde{u}_{i}=0, C_{p(.), \omega}$-quasi-everywhere in $X \backslash E$.

Since $M_{p(.), \mu}^{1}(X, \omega)$ is a Banach space, there is a function $u \in M_{p(.), \mu}^{1}(X, \omega)$ such that $\tilde{u}_{i} \rightarrow u$ in $M_{p(.), \mu}^{1}(X, \omega)$ as $i \rightarrow \infty$. By theorem 4.4, $u$ is $C_{p(.), \omega}$-quasicontinuous and by theorem 4.5 we have $u=0 C_{p(.)}$-quasi-everywhere in $X \backslash E$. Thus $u \in B_{p(.)}^{1,0}(E, \omega)$ and the spaces $B_{p(.)}^{1,0}(E, \omega)$ is complete.

Corollary 5.1. The space $B_{p(.)}^{1,0}(E, \omega)$ is reflexive.

Proof. The space $M_{p(.), \mu}^{1}(X, \omega)$ is reflexive Banach, by theorem5.1 the space $B_{p(.)}^{1,0}(E, \omega)$ is closed, and therefore $B_{p(.)}^{1,0}(E, \omega)$ is reflexive.

Lemma 5.1. Let $u \in B_{p(.),}^{1,0}(E, \omega)$ and $v \in M_{p(.), \mu}^{1}(X, \omega)$ are bounded functions. If $v$ is $C_{p(.), \omega}$-quasicontinuous, then $u v \in B_{p(.)}^{1,0}(E, \omega)$.

Proof. Let $\tilde{u} \in M_{p(.), \mu}^{1}(X, \omega)$ be the $C_{p(.), \omega}$-quasicontinuous representative of $u$. we have $\tilde{u} v$ is $C_{p(.), \omega}$-quasicontinuous in $X$. Let $D=\{x \in X \backslash E: \tilde{u} v \neq 0\}$, we have $D=G \cup H$ where $G=\{x \in X \backslash E: \tilde{u} \neq 0\}$ and $H=\{x \in X \backslash E: v=\infty\}$. It obvious that $C_{p(.), \omega}(G)=0$ and by theorem4.3 we have $C_{p(.), \omega}(H)=0$, thus $C_{p(.), \omega}(D)=0$ and $\tilde{u} v=0 \quad C_{p(.), \omega}$-quasi-everywhere in $X \backslash E$. Since $\tilde{u} v=u v$ almost everywhere in $E$, then $u v \in B_{p(.)}^{1,0}(E, \omega)$.

Theorem 5.2. Let $G \subset E$ such that $C_{p(.), \omega}(G)=0$, we have

$$
B_{p(.)}^{1,0}(E, \omega)=B_{p(.)}^{1,0}(E \backslash G, \omega) .
$$

Proof. It obvious that $B_{p(.)}^{1,0}(E \backslash G, \omega) \subset B_{p(.)}^{1,0}(E, \omega)$. Let $u \in B_{p(.)}^{1,0}(E, \omega)$ there is a $C_{p(.), \omega}$-quasicontinuous function $\tilde{u} \in M_{p(.), u}^{1}(X, \omega)$ such that $\tilde{u}=u$ almost everywhere in $E$ and $\tilde{u}=0 \quad C_{p(.), \omega}$-quasi-everywhere in $X \backslash E$. Since $C_{p(.), \omega}(G)=0$, we have $\tilde{u}=0 \quad C_{p(.), \omega}$-quasi-everywhere in $X \backslash(E \backslash G)$. Thus

$$
u \in B_{p(.)}^{1,0}(E \backslash G, \omega) .
$$

Remark 5.2. If $C_{p(.), \omega}(\partial E)=0$, then $B_{p(.)}^{1,0}($ int $E, \omega)=B_{p(.), \omega}^{1,0}(\bar{E}, \omega)$. 
Theorem 5.3. Let $O$ be an open subset of $X$. Then $B_{p(.)}^{1,0}(E, \omega)=B_{p(.)}^{1,0}(E \backslash O, \omega)$. if and only if $C_{p(.), \omega}(E \cap O)=0$.

Proof. It is the same as that given in [14], Theorem 4.8 .

\section{The Dirichlet Energy Integral Minimisers}

In this section, $(X, d)$ is a metric space with finite diameter, $\left(\operatorname{diam}(X)=\sup _{x, y \in X} d(x, y)<\infty\right)$ and equipped with a finite, positive Borel regular outer, atomless measure $\mu$. The triplet $(X, d, \mu)$ will be fixed in the sequel and will be denoted by $X$.

$\omega$ is a positive measurable and finite function.

$p: X \longrightarrow[1,+\infty)$ is a variable exponent such that $1<p^{-}<p^{+}<\infty$.

Definition 6.1. Let $h \in M_{p(.), \mu}^{1}(X, \omega)$. For all $u \in B_{p(.)}^{1,0}(E, \omega)$ we put

$$
I_{E, h}^{p(.), \omega}(u)=\int_{E}\left(|u(x)|^{p(x)}+\omega(x)\left(\left(g_{u}(x)\right)^{p(x)}+\left(g_{h}(x)\right)^{p(x)}\right)\right) d \mu(x),
$$

$I_{E, h}^{p(.), \omega}$ is call the energy operator corresponding to the boundary value function $u$.

Lemma 6.1. [?] Let E be a reflexive Banach space. If $I: E \longrightarrow \mathbb{R}$ is lower semicontinuous and coercive operator, then there is an element in $E$ that minimizes $I$.

Theorem 6.1. There exists a function $u \in B_{p(.)}^{1,0}(E, \omega)$ such that

$$
I_{E, h}^{p(.), \omega}(u)=\inf _{v \in B_{p(.)}^{1,0}(E, \omega)} I_{\Omega, h}^{p(.), \omega}(v) .
$$

Proof. We have $B_{p(.)}^{1,0}(E, \omega)$ is a reflexive Banach space.

Since $x \longrightarrow x^{p}$ is convex for every fixed $1<p<\infty$, then $I_{E, h}^{p(.), \omega}$ is convex.

Let $\left(u_{i}\right)$ be a sequence of functions in $B_{p(.)}^{1,0}(E, \omega)$ converging to $u \in B_{p(.)}^{1,0}(E, \omega)$. Then $u_{i} \longrightarrow u$ in $L^{p(x)}(X)$ and $g_{u_{i}} \longrightarrow g_{u}$ in $L^{p(x)}(X, \omega)$, thus $u_{i} \longrightarrow u$ and $g_{u_{i}} \longrightarrow g_{u} \mu$ almost every where in $X$ and by Fatou's theorem we have $I_{\Omega, h}^{p(.), \omega}$ is lower semicontinuous.

If $\|u\|_{B_{p(.)}^{1,0}(E, \omega)} \longrightarrow \infty$ then $I_{\Omega, h}^{\varphi}(u) \longrightarrow \infty$, and therefore the operator $I_{\Omega, h}^{p(.), \omega}$ is coercive, and the theorem follows by lemma 6.1 .

\section{References}

[1] Adams, D.R. and Hedberg, L.I. Function spaces and potential theory, Springer (1999).

[2] Aissaoui, N. and Benkirane, A. Capacité dans les epaces d'Orlicz, Ann. Sci. Math. Québec 18 (1) (1994), 1 - 23.

[3] Aissaoui, N. and Benkirane, A. Potentiel non lineaire dans les espaces d'Orlicz. Ann. Sci. Math. Québec 18 (2) (1994), $105-118$.

[4] N. Aissaoui, Strongly nonlineaire potentiel, Abstract and Applied Analysis, 2002, 357-374.

[5] David Cruz-Uribe , Lars Diening, HÄSTÖ. The maximal operator on weighted variable Lebesgue spaces, Georgian Mathematical Journal 15(4) ̊ January (2008).

[6] Diening, L., Harjulehto, P., HÄSTÖ, P. and Rudicka, M. Lebesgue and Sobolev spaces with variable exponents. Lecture Notes in Mathematics, vol, 2017. Springer, Berlin (2011).

[7] P. Hajlasz, Sobolev space on arbitry metric space, Kluwer academic publishers, 1996, 403-415.

[8] M.C. Hassib, Y. Akdim, N. Aissaoui and. Musielak-Orlicz-Sobolev spaces on arbitrary metric space. Commentationes Mathematicae. vol. 
[9] P. Harjulehto, P. HÄSTÖ and M. Pere, Variable exponent Lebesgue spaces on metric spaces, the HardyLittlewood maximal operator, Real Anal. Exchange 30 (2005), no. 1, 87-104.

[10] Ismail Aydin. Journal of Function Spaces and Applications. Volume 2012, 17 pages.

[11] J. Kinnunen and O.Martio, The Soboleve capacity on metric spaces, Annales Academic Scientiarum Fennicae, 1996, 367-382.

[12] E. J. McShane, Extension of range of functions, Bull. Am. Math. Soc, 1934, 837-842.

[13] Ismail Aydin. Journal of Function Spaces and Applications. Volume 2012, 17 pages.

[14] T. Kilpeläinen, J. Kinnunen, and O. Martio, Sobolev spaces with zero boundary values on metric spaces, Potential Analysis, 12 (2000), 233-247.

[15] Petteri Harjulehto, Peter Hasto and Mikko Pere. Variable exponent Sobolev spaces on metric measure spaces, Funct. Approx. Comment. Math.36, 2006, 79-94.

[16] Takao Ohno and Tetsu Shimomura. Musielak-Orlicz-Sobolev spaces on metric measure spaces. Czechoslovak mathematical journal, Volume 65, (2015), Issue 2, pp 435-474. 